\title{
The Relationship between Social Skills and Sports Activities among Chinese College Students
}

\author{
Lu Lv, Kazushi Takami \\ Graduate School of Human Development and Environment, Kobe University, Kobe, Japan \\ Email: roro@stu.kobe-u.ac.jp
}

Received 24 February 2015; accepted 11 March 2015; published 16 March 2015

Copyright (C) 2015 by authors and Scientific Research Publishing Inc.

This work is licensed under the Creative Commons Attribution International License (CC BY). http://creativecommons.org/licenses/by/4.0/

\section{(c) (i) Open Access}

\begin{abstract}
The purpose of this study was to understand the current state of social skills among Chinese student, especially focused on the students who participated in sports clubs and took physical exercise regularly, and subsequently conducted a new multi-index model further to explore the relationship between social skills and sports activities. 3000 college students were investigated by two scales named revised Chinese university-student social skills inventor (RChUSSI) and the Chinese college students' version of Buss-Perry aggression questionnaire (CC-BPAQ), and resulted in $2543(84.7 \%)$ samples return. By the help of analysis of variance and multivariate analysis of covariance, a multi-index model was conducted and examined. This study reported that the period of primary and secondary school might be the most productive years of human achieving and training social skills. Taking sports activities facilitated learning and improving social skills. Thus, primary and secondary school students are encouraged to have sports activities, which are good for their physical and mental health.
\end{abstract}

\section{Keywords}

Social Skills, Sports Activities, Chinese College Students, Sports Club Members

\section{Introduction}

During the interaction and communication processing with others, we use some skills, both verbally and nonverbally, to help us to understand them, meet potential target, and consequently develop many relative interpersonal behaviors. In the whole processing, we also can understand and control our own emotion which enables us to have smooth and comfortable interactions with any one, on any topic. These skills were defined as social 
skills (Aikawa, 2009). Social skills are entirely involved in our life throughout as life skills we use every day. For instance, they actually impacted on school achievement and desire for leaning when we are in primary and secondary school (Kim et al., 1968; Feldhusen et al., 1970). Moreover, social skills have been suggested to be associated with juveniles’ aggression (Matsushita \& Muramatu, 2006), bullying (Shimizu et al., 2009), school adjustment (Otsui, 2011), loneliness and psychology health (Aikawa et al., 1993) and even to occupational stress (Tanaka, 2009). Anyway, it is clearly known that social skills are crucial for building great relationship. For its importance, it is significant to further study on social skills for relieving and resolving the tension appeared in interaction and many social problems.

It has been shown that a healthy and harmonious interpersonal relationship produced a great effect on subjective well-being for college students (Zhang et al., 2009). Similarly, Hong et al. (2013) further explored the relationship between group attachment and interpersonal relationship, and reported that interpersonal disturbance troubled almost of college students in China (Hong et al., 2013). These evidences suggested there is an urgent need for improving college students' social skills to enable them to communicate effectively with others. In addition, Ishikura (1999) performed study in freshmen and found that physical education facilitated the interaction and communication with others (Ishikura, 1999), while Shimamoto (2007) and Ishii suggested there was a positive correlation between exercise experiences and improvement of social skills (Shimamoto \& Ishii, 2007). Furthermore, Tokunaga (2002) studied correlates of sports and social skills among college students. Participants were classified into three groups. The first group was consisting of who joined sports clubs, while the second one for who entered into non-sports clubs, such as music club. And the college students didn't join any clubs were defined as the third group. The result showed that the first group showed better social skills than the second and third (Tokunaga, 2002). As above mentioned, obviously, the one who having physical exercise has greater social skills than the others. That means taking physical exercise actively has a positive effect on acquiring social skills.

In China, the research about undergraduates' social skills is still in its early stage. Lv and Takami (2014) made a survey among 1916 Chinese college students and suggested that club activities in school played a major role in improving social skills among college students (Lv \& Takami, 2014). In the above study, however, the subjects were not classified in detail to explore the influence on social skills derived from sports. Hereby, the present study selected college students as subjects, especially focused on who participated in sports clubs and took physical exercise regularly, and specifically, aimed to understand the current state of their social skills. Furthermore, we attempted to explore how sports activities affect college students' social skills.

\section{Methods}

\subsection{Subjects}

Over a period of 3 months from April to June in 2011, by convenience sampling, 9 comprehensive universities among following cities: Beijing, Shanghai, Chongqing, Chengdu, Changsha, Hangzhou, Nanjing, Dalian and Nanchang were chosen based on administrative division, and then drew random samples from these universities. Questionnaire was conducted among 3000 college students which resulted in 2543 (84.7\%) samples return. The effective recovery of 2347 was carried onto next work. In other word, 2347 participants (male $=1141$, female $=$ 1206) were recruited in present study, normally aged 16 - 29 (20 \pm 1.38 ), which included 897 freshmen, 933 sophomores, 442 juniors and 75 seniors.

Among them, there were 780 sports club members (SCMs), 820 non-sports club members (NSCMs) and 747 non-members (NMs) who didn't participate in any clubs. However, this is not so ideal. To get who taking physical exercises regularly, according classification and statistical criteria of sports population (Xiao \& Fang, 2005), if selected SCMs in above work don't meet the condition "taking exercise $>=$ three times per week and $>=30$ minutes every time", we would remove them from SCMs group. After a careful choice, only 292 SCMs were left. Finally, 1859 samples (870 males and 989 females) were taken into statistical analysis, including 732 freshmen, 758 sophomores, 308 juniors and 61 seniors. Because of less number of seniors, we regarded juniors and seniors as one group to further analysis.

\subsection{Measurements}

\subsubsection{Revised Chinese University-Student Social Skills Inventor (RChUSSI)}

The RChUSSI scale was developed and used to measure college students' social skills (Lv \& Takami, 2014). 
This scale is composed of three sub-scales (34 items), "thoughtful”, "sociability” and "social shyness” (reverse scoring), where participants rank certain statement along a 5 point continuum from "extremely uncharacteristic for me" to "extremely characteristic for me". This scale has been tested and validated successfully for Chinese college students (Cronbah's $\alpha$ ranged from $0.86-0.93$, test-retest reliability coefficient $=0.086$ ). For this study, we used this scale to examine the social skills of SCMs, NSCMs and NMs.

\subsubsection{The Chinese College Students' Version of Buss-Perry Aggression Questionnaire (CC-BPAQ)}

The personality factors and health outcomes was measured using the Buss-Perry Aggression Questionnaire by Buss and Perry, 1992. Lv et al. (2013) developed the Chinese College Students' Version of Buss-Perry Aggression Questionnaire (Lv et al., 2013), composed of 4 subscales (22 items), namely "hostility", "physical aggression", "impulsivity" and "anger proneness". The Cronbah's $\alpha$ for the 4 subscales was $0.73-0.85$, while the testretest reliability coefficient was 0.91 , which suggested the CC-BPAQ scale is reliable and valid. Thereby, in this study CC-BPAQ was used to measure the aggression of SCMs NSCMs and NMs.

\subsubsection{A Multi-Index Model}

This study not only tested differences of social skills between sports participants and non-sports participants, but also explored and analyzed how sports activities affect social skill using multivariate analysis of covariance (MANCOVA). Referring to previous works by Aoki (2006) and Lv et al. (2014), we developed a multi-index model to obtain the link between sports activities and social skills (Figure 1). In the model, sports activates was consist of "athlete level", "how many months", "how long per week". Social skills were consist of "thoughtful", "sociality”, "social shyness", while aggression was consist of "hostility”, "physical aggression”, "impulsivity" and "anger proneness". Mugno (1985) and Sheids (1995) reported that the athletes showed high aggression (Mugno \& Feltz, 1985; Shields \& Bredemeier, 1995). Moreover, Hao and his coworkers (2008) reported the aggression of athletes was higher than college students (Hao et al., 2008). From these previous evidences, it is known that the sports activities may promote aggression, and the aggression had negative impact on social skills at some degree (Ando \& Yoshimura, 2003). Thus, in this study, aggression was selected as intermediate factor. We assumed that sports activities have an influence on social skills directly and/or indirectly.

\subsection{Procedures}

In this study, the survey was assessed by anonymous questionnaires. We sent questionnaires to the student organizations among 9 universities we selected and entrusted them with issuing and collecting questionnaires. Statistical analysis was performed on SPSS 21 and AMOS18.

\section{Results}

\subsection{The Current State of Social Skills}

\subsubsection{The Differences of Social Skills between SCMs, NSCMs and NMs with Gender}

The results of RChUSSI scales were presented in Table 1, with stratification by gender, SCMs, NSCMs and NMs. The review of statistical date (Table 1 ) showed that groups (participated sports club, club or not) had a significant main effect in following factors: "thoughtful" $(F(2,1853)=36.46, p<0.01)$, "sociality" $(F(2,1853)=$ 5.28, $p<0.01)$ and "social shyness" $F(2,1853)=28.52, p<0.01)$, while gender had main effect in "thoughtful" $(F(1,1853)=4.32, p<0.05)$ and "sociality" $(F(1,1853)=11.37, p<0.01)$. And there was no interaction between groups and gender. Compared in pairs (Bonferroni), SCMs and NSCMs showed higher scores than NMs for "thoughtful" $\left(\mathrm{M}_{\mathrm{SCMs}}-\mathrm{M}_{\mathrm{NM}}=6.17, \mathrm{M}_{\mathrm{NSCMs}}-\mathrm{M}_{\mathrm{NMs}}=4.37\right)$ and "social shyness" $\left(\mathrm{M}_{\mathrm{SCMs}}-\mathrm{M}_{\mathrm{NMs}}=2.45\right.$, $\left.\mathrm{M}_{\mathrm{NSCMs}}-\mathrm{M}_{\mathrm{NMs}}=1.91\right)$; For "sociality", $\mathrm{SCMs}$ showed much higher score than NSCMs and NMs $\left(\mathrm{M}_{\mathrm{SCMs}}-\mathrm{M}_{\mathrm{NMs}}=\right.$ $\left.1.94, \mathrm{M}_{\mathrm{SCMs}}-\mathrm{M}_{\mathrm{NSCMs}}=1.71\right)$.

\subsubsection{The Differences of Social Skills between SCMs, NSCMs and NMs with School Year}

Meanwhile, we also tested whether there were some differences between different school years. Defined group and school year as independent variances, the results of RChUSSI demonstrated group had main effect in "thoughtful" $(F(2,1850)=34.23, p<0.01)$, "sociality" $(F(2,1850)=9.89, p<0.01)$ and "social shyness" $(F(2,1850)=26.42, p<0.01)$. In addition, there was an interaction effect of group and school year in "sociality" $(F(4,1850)=3.10, p<0.05)$. 


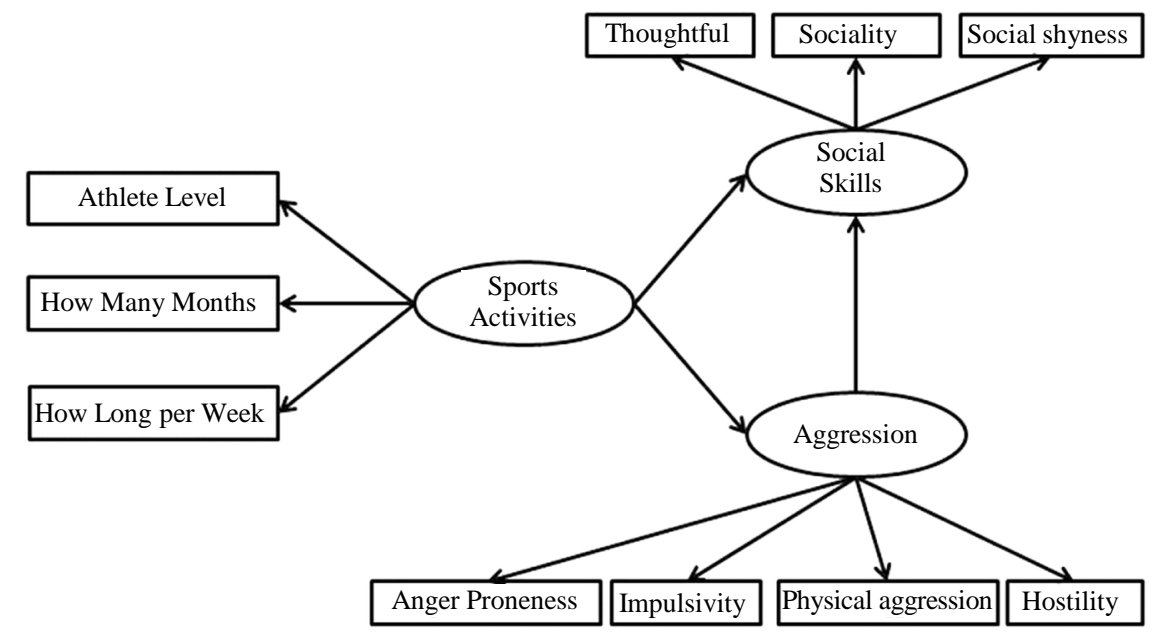

Figure 1. A multi-index model.

Table 1. The differences between groups and gender by RChUSSI.

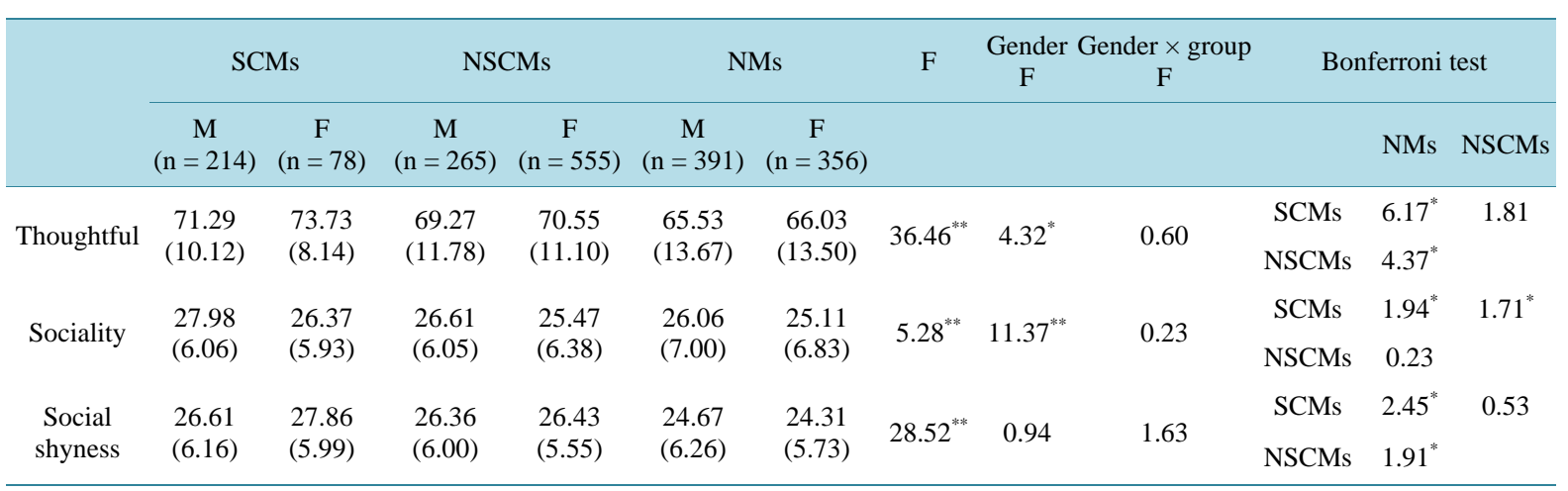

Note: This table presented the mean value of every factor among different group. ( ) indicates the standard deviation of every factor among different group. SCMs: sports club members; NSCMs: non-sports club members; NMs: non-members of any club. ${ }^{*} p<0.05,{ }^{* *} p<0.01$.

To show the change of social skills with school year increasing intuitively, we presented them by line charts (Figures 2-4). These figures revealed that the social skills' score of freshmen who participated sports club was the highest measured by RChUSSI scale, but it was decreasing with school year increasing. For NSCMs and NMs, the score was both increasing with school year increasing but the former is better than the latter. In addition, NSCMs showed higher social skills than SCMs when they were juniors and/or seniors.

\subsection{The Effects of Sports Activities on Social Skills}

To clarify the relevance between sports activities and social skills, this study conducted a multi-index model compose of 3 concepts, "sports activities", "social skills" and "aggression”, totally 10 observation variances (Figure 1), which was analyzed and modified using MANCOVA. Figure 1 as defined earlier presented the initial model. Admittedly, initial model showed less fitting with data. Thus, we modified it by a correction factory and then we obtained a new model as Figure 5 shown, which has good coherence with data (GFI $=0.93$, AGFI $=0.87, \mathrm{CFI}=0.84, \mathrm{RMSEA}=0.09$ ). Furthermore, to examine whether there are any changes among the $10 \mathrm{ob}-$ servation variances in this model affected by gender, we used the method of multiple group structural equation modeling and found a high degree of fitting with female and male samples for this model (GFI $=0.90$, AGFI $=$ 0.82 , CFI $=0.82$, RMSEA $=0.85$ ). That means there was no difference of gender.

As Figure 5 presented, sports activities had a weak effect on aggression in our model. Meanwhile aggress affected social skills negatively at some degree. Which means the social skill of who shows higher aggression is lower. In other words, sports activities barely impacted on social skills indirectly the intermediate factor "aggression". 


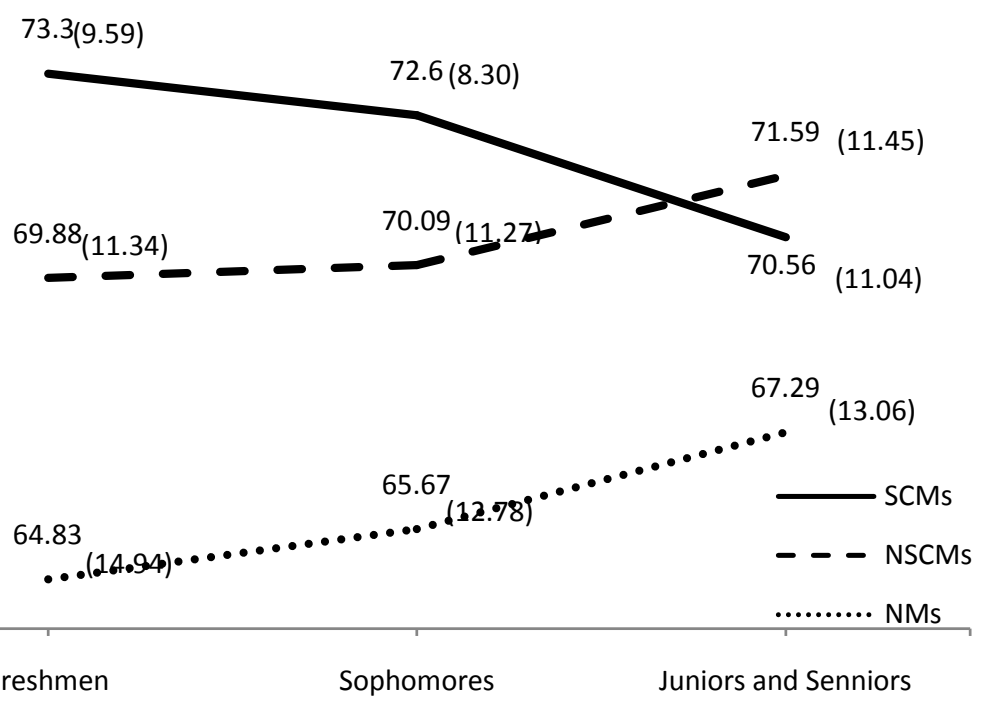

Figure 2. “Thoughtful”.

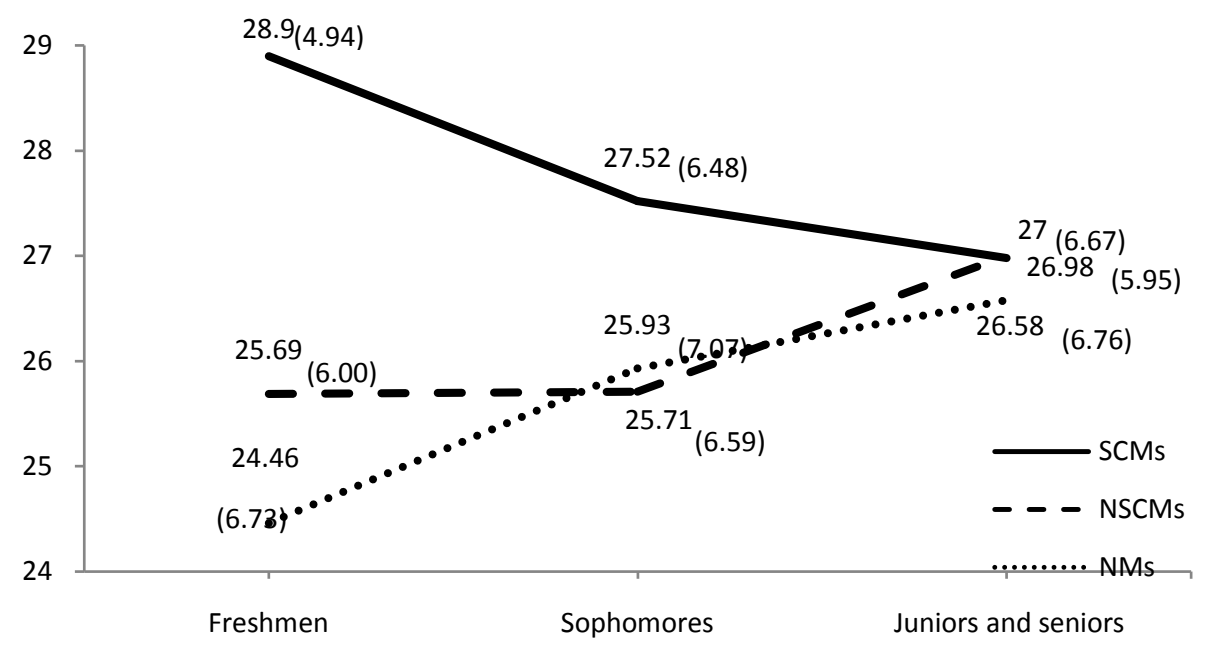

Figure 3. "Sociality”.

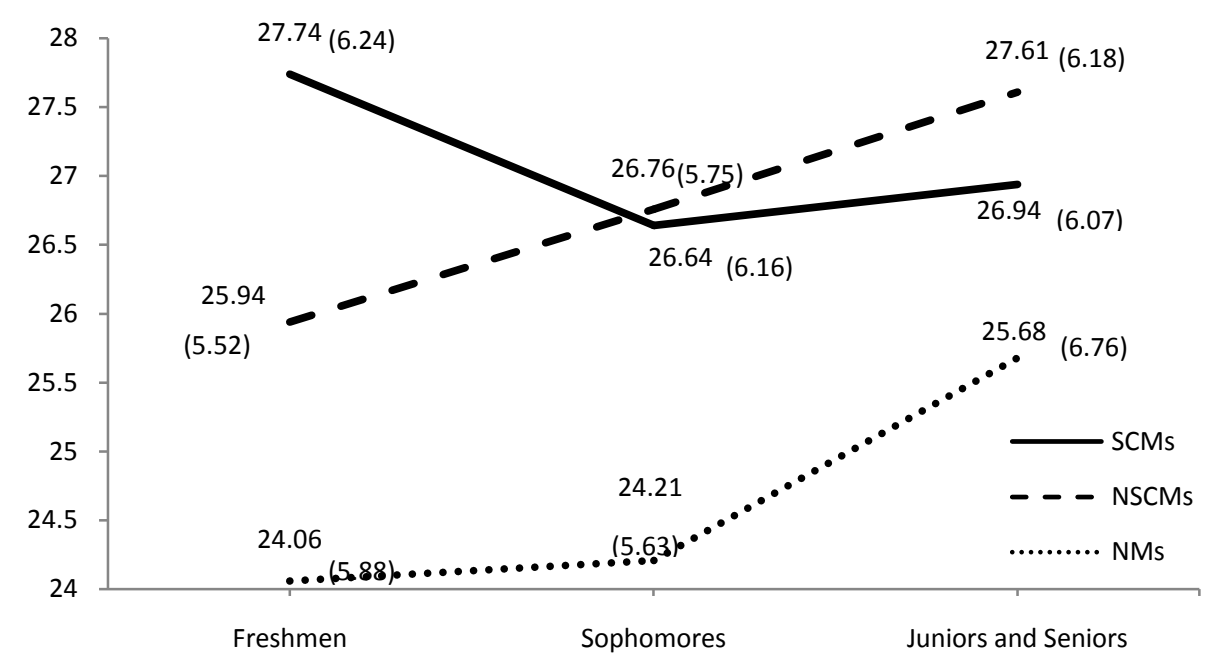

Figure 4. "Social shyness”. 


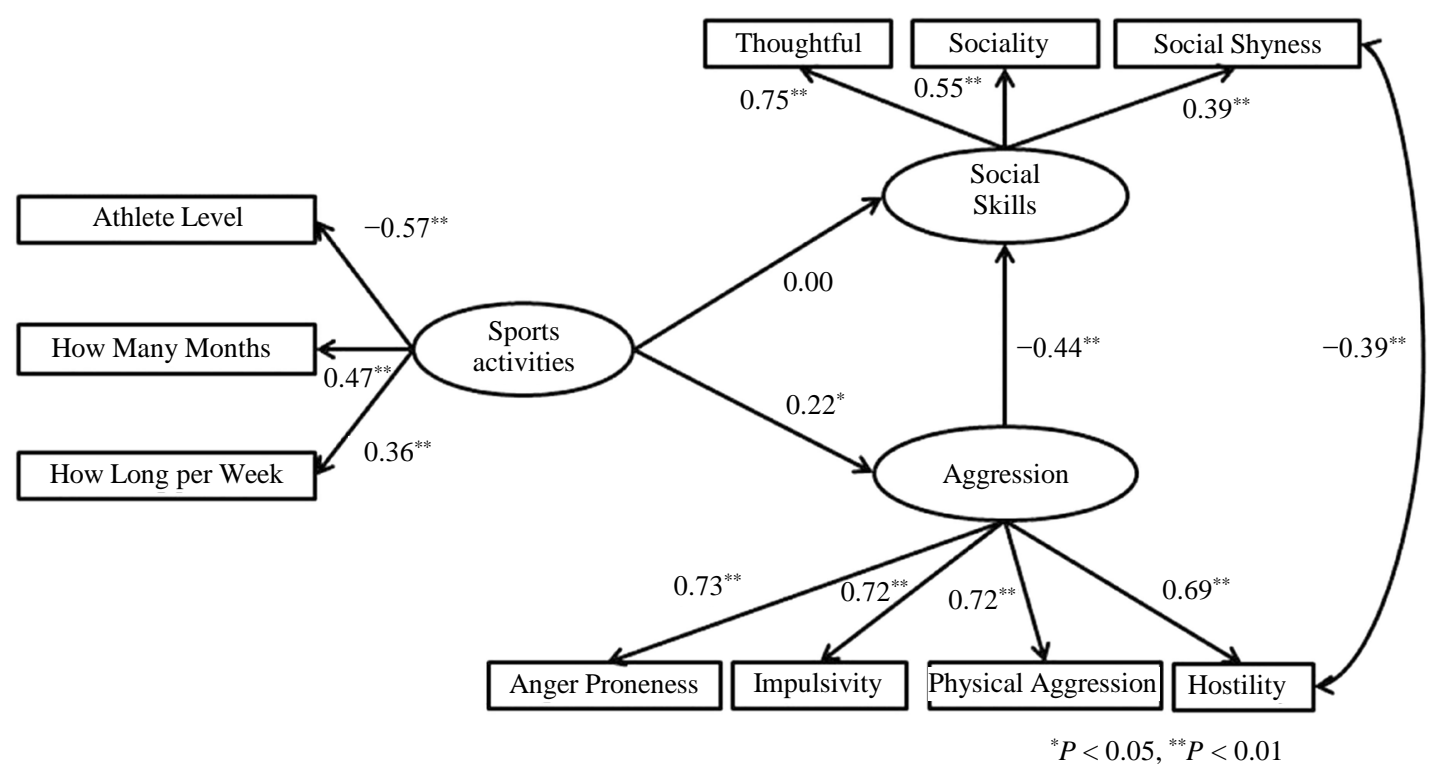

Figure 5. The modified multi-index model.

\section{Discussion}

For "thoughtful", the present study showed the female is higher than male students. While, for "sociality", male is higher than female college students. After classified and compared with each other, the social skills of SCMs and NSCMs were better than NM. This was in agreement with the previous study that there were some positive factors for improving social skills in club activities (Lv \& Takami, 2014).

In addition, considered by school year, among SCMs, the social skills' score of freshmen was the best, and the score seemed inversely proportional to the school year. But anyway, the SCMs always showed better social skills than another. Thus, firstly, we concluded that the SCMs mainly obtained their social skills before entering into university; After entering into university, although the social skills of SCMs were going down with school year increasing, it was always in a high level.

The previous works by Fan and his coworkers (2000) reported lack of the number of places for physical activities was resulted in college students mainly took sports exercises in campus while primary and secondary school students in free and public activity places nearby home (Fan et al., 2000). For this reason, the primary and secondary school students have more chances to communicate with another during physical exercises processing and that benefits achievement and improvement of social skills. Evidence from Yamamoto (1998) showed the disorganized sports activities were effective and positive for children's social skills. Due to the sports lovers trained their social skills effectively with having physical exercises when they were primary and/or secondary school students, the present study found the freshmen show the best social skills.

Meanwhile, this study found that after entering into university, the social skills of SCMs was decreasing with school year increasing. Shimamoto and Ishii (2007) demonstrated that the training condition and the coach have valuable influence on social skills of sports club participants (Shimamoto \& Ishii, 2007). Compared with university sports organizations, the sports clubs provided more opportunities to achieve and improve social skills. Moreover, the sports activities and programs offered by university sports organization are usually held at a fixed time and location, even with fixed partners, which reduced the chance of training social skills.

To clarify how sports activities affect social skills, we selected aggression as an intermediate factor and then processed MANCOVA. As Figure 5 represented, sports activities didn't affect social skills directly in our model, and sports activities had a weak influence on aggress, which was in line with the previous studies (Lv et al., 2014). We conduced that the sports activities had an effect on social skills in some ways except aggression.

The present findings were consistent with some previous studies but simultaneously it was admitted that there were some limitations in our study. In this work, we just compared subjects by the cross-sectional analysis. It would be better to understand the change of one's social skills over long periods of time by the longitudinal analysis. 


\section{Conclusion}

The present study used the RChUSSI and CC-BPAQ scales to observe the social skills state of sports club members among Chinese college students and tried to build a new multi-index model to explore the relationship among sports activities, aggression and social skills. As result, we found that the period of primary and secondary school may be the most productive years of human achieving and training social skills. And, taking sports activities will provide more chances for students learning and improving social skills. Thus, we encourage the students of primary and secondary school to take physical exercises, which are beneficial to physical and mental health.

\section{References}

Aikawa, T. et al. (1993). A Study on the Interpersonality Behaviors of Lonely University Students: The Relationship between the Self-Reported Loneliness and Social Skills Deficits. The Japanese Society of Social Psychology, 8, 44-55.

Aikawa, T. (2009). The New Version of Communication Skills_Psychology of Social Skills. Tokyo: Science.

Ando, A., \& Yoshimura, H. (2003). Aggression and Social Skills. Bulletin of Education Science, Kyoto Women's University, 43, 131-149.

Aoki, K., \& Matsumoto, K. (2006). Aggression in High School Athletes and Its Correlates. Bulletin of the Faculty of Social Welfare, Yamaguchi Prefectural University, 12, 1-15.

Fan, L. R. et al. (2000). A Nationwide Survey on Students’ Physical Activities after School. Sport Science, 20, 7-11.

Feldhusen, J. F., Thurston, J. R., \& Benning, J. J. (1970). Longitudinal Analysis of Classroom Behavior and School Achievement. Journal of Experimental Education, 38, 4-10. http://dx.doi.org/10.1080/00220973.1970.11011207

Hong, Y. P., Jiang, X. X., \& Zhou, X. G. (2013). Relationship between Group Attachment, Interpersonal Relationship and Class Cohesion among College Student. China Journal of Health Psychology, 21, 919-921.

Ishikura, T. (1999). The Change of Freshmen's Social Skills Who Got the Sports Skill Education among D University. Doshisha Journal of Health and Sport Science, 38, 23-44.

Kim, Y., Andson, H., \& Bashaw, W. L. (1968). Social Maturity Achievenment and Basic Ability. Educational and Psychological Measurement, 28, 535-543. http://dx.doi.org/10.1177/001316446802800240

Lv, L., Takami, K., Dong, D., Wong, L., \& Wang, X. (2013). Development of the Chinese College Students'. Version of Buss-Purry Aggression Questionnaire. Chinese Mental Health Journal, 27, 378-383.

Lv, L., \& Takami, K. (2014). The Development of the Revised Chinese University-Students Social Skill Inventory and the Current State of Social Skills. China Journal of Health Psychology, 22, 884-889.

Lv, L., Mei, X. L., Lu, F., \& Takami, K. (2014). Influence of Sports Activities on Undergraduates’ Aggression: An Analysis on Intermediary Function of Shyness. Journal of Beijing Sport University, 37, 70-75.

Matsushita, K., \& Muramatsu, J. (2006). A Study on Aggression, Self-Esteem and Social Skill among Junior High School Students. Tokai Journal of School Health, 30, 47-60.

Otsui, K. (2011). Social Skills, Social Support and High School Student Adjustment: A Comparison with Truants. Bulletin of the Faculty of Sociology Science, Kinki University, 1, 23-33.

Shimizu, K. et al. (2009). The Relationship between Social Skills and Aggression, and Aggression Susceptibility among Junior High School Students. Tokai Journal of School Health, 33, 53-67.

Shimamoto, K., \& Ishii, M. (2007). Construction of a Causal Model for the Relationship Sport Experience and Life Skills. Sport Psychology Research, 34, 1-9.

Tanaka, K. (2009). Social Skills and Job Stress. Tokyo: Koyoshobo.

Xiao, H. Y., \& Fang, L. (2005). Concept, Classification and Statistical Criteria of Sports Population. Sport Science Research, 26, 7-10.

Yamamoto, K. (1998). A Cosmology of Child's Sport-Possibility and Limitation of Building Social Skills. Bulletin of the Faculty of Education, Kagoshina University (Cultural and Social Science), 50, 185-192.

Zhang, C., Ban, L. M., \& Li, C. N. (2009). Relationship between Interpersonal Disturbances and Subjective Well-Being of College Students. China Journal of Health Psychology, 17, 1466-1468. 